\title{
An Ophthalmology Resident-Led Quality Improvement Initiative to Decrease the Incidence of Perioperative Corneal Injury
}

\author{
Amanda L. Ely, MD ${ }^{10} \quad$ Mark Goerlitz-Jessen, MD ${ }^{2}$ Ingrid U. Scott, MD, MPH ${ }^{1,3}$ Erik Lehman, MS 3 \\ Tabassum Ali, MD ${ }^{4}$ Denise Kerchner, $\mathrm{MD}^{5}$ David Liang, $\mathrm{MD}^{6}$
}

\footnotetext{
${ }^{1}$ Department of Ophthalmology, Penn State College of Medicine, Hershey, Pennsylvania

2 Department of Ophthalmology, Duke University Medical Center, Durham, North Carolina

${ }^{3}$ Department of Public Health Sciences, Penn State College of

Medicine, Hershey, Pennsylvania

${ }^{4}$ Delaware Ophthalmology Consultants, Wilmington, Delaware

${ }^{5}$ Elmwood Eye Center, York, Pennsylvania

${ }^{6}$ Department of Ophthalmology, Central Texas Veterans

Administration, Temple, Texas
}

J Acad Ophthalmol 2019;11:e49-e53.

\begin{abstract}
Address for correspondence Amanda L. Ely, MD, Penn State Eye Center, 500 University Drive UPC 1, Suite 800, HU19, Hershey, PA 17033 (e-mail: aely@pennstatehealth.psu.edu).
\end{abstract}

\begin{abstract}
Keywords

- perioperative corneal injury

- corneal abrasion

- exposure keratopathy

- quality improvement initiative

Objective This article evaluates the effectiveness of an ophthalmology resident-led quality improvement (QI) initiative to decrease the incidence of perioperative corneal injury at an academic medical center

Design Retrospective chart review.

Methods A retrospective chart review was conducted of all surgical cases performed 6 months prior to, and 6 months after, implementation of an ophthalmology residentled QI initiative at an academic medical center. The QI initiative (which focused on perioperative corneal injury awareness, understanding of risk factors, and presentation of an algorithm designed to prevent perioperative corneal injury) consisted of a lecture and distribution of educational materials to anesthesia providers. Data collected through the chart review included type of surgical case, presence of diabetes mellitus or thyroid disease, patient age and gender, patient positioning (supine, prone, or lateral), level of anesthesia provider training, length of surgical case, surgical service, type of anesthesia, and type (if any) of perioperative eye injury. The rates of perioperative corneal injury pre- versus post-initiative were compared.

Results The rates of perioperative corneal injury pre- and post-initiative were 3.7 and 1.9 per 1,000 , respectively $(p=0.012)$. Significant risk factors for perioperative corneal injury include longer duration of surgery (odds ratio $[O R] 90-180$ vs. $<90$ minutes $=4.18,95 \%$ confidence interval $[\mathrm{Cl}] 1.43-12.18 ; \mathrm{OR}>180$ vs. $<90$ minutes $=8.56,95 \% \mathrm{Cl} 3.01-24.32$; OR $>180$ vs. $90-180=2.05,95 \% \mathrm{Cl} 1.17-3.58$ ), patient position lateral $>$ prone $>$ supine (OR prone vs. lateral $=0.25,95 \% \mathrm{Cl} 0.09-0.67$; OR supine vs. lateral $=0.13,95 \% \mathrm{Cl}$ $0.07-0.23)$, nonhead and neck surgeries $(\mathrm{OR}=0.32,95 \% \mathrm{Cl} 0.11-0.87)$, and surgery performed under the general surgery service (OR general surgery service vs. other subspecialty services $=6.50,95 \% \mathrm{Cl} 2.39-24.76)$.
\end{abstract}

received

August 22, 2019 accepted after revision October 16, 2019
DOI https://doi.org/

10.1055/s-0039-3400545. ISSN 2475-4757.
Copyright $\odot 2019$ by Thieme Medical Publishers, Inc., 333 Seventh Avenue, New York, NY 10001, USA. Tel: +1(212) 584-4662.
License terms

(c) (1) $\ominus$ (\$) 
Conclusions An ophthalmology resident-led QI initiative consisting of educating anesthesia providers was associated with a significant decrease in the rate of perioperative corneal injury.

Corneal injury is the most common perioperative ocular injury. ${ }^{1}$ While data on perioperative corneal injury awareness is highly published in the anesthesia literature, there are few reports in the ophthalmology literature. The American Society of Anesthesiologists (ASA) Closed Claims Project reported that, prior to 1990 , eye injuries were responsible for $3 \%$ of all claims and, of these, $35 \%$ were corneal injuries. ${ }^{2} \mathrm{~A}$ 2014 ASA publication reports that the incidence of perioperative corneal injury declined from $31 \%$ before 1995 to $18 \%$ in the period 1995 to $2011 .^{2,3}$

The most common mechanisms of perioperative corneal injury include direct trauma to the corneal epithelium (corneal abrasion), corneal drying (exposure keratitis), and chemical exposure (toxic keratopathy). ${ }^{2}$ The eye may be injured by direct trauma either from tape used to close the eyelids, the face mask, the anesthesia provider's watch strap, name badge, or laryngoscope during intubation, drapes during surgical preparation, or the pulse oximeter probe upon patient emergence from anesthesia. ${ }^{2,4,5}$ Alcohol and chlorhexidine-containing surgical preparation solutions have been shown to cause corneal epithelial, as well as endothelial, disruption. ${ }^{6}$ Anesthesia itself also poses a direct threat to the cornea, as it decreases the production and stability of tears, abolishes the normal blink reflex, and abolishes the normal Bell's phenomenon that innately protects the cornea during sleep. ${ }^{3}$ Patients with anatomical variations such as lagophthalmos or exophthalmos are at increased risk of perioperative corneal injuries. ${ }^{1,3}$ Additional reported risk factors for perioperative corneal injury include prone and lateral positioning, head and neck surgery, prolonged surgery (> 90 minutes), early practitioner training level, presence of diabetes mellitus or thyroid disease, intraoperative sustained hypotension, and anemia. ${ }^{7-14}$

A computerized search of the PubMed database reveals four academic institutions that have published in the anesthesia literature the incidence of perioperative corneal injury at their sites. The incidence of perioperative corneal injury was reported to be $0.15 \%$ in 2005 and $0.33 \%$ between 2011 and 2017 at the Mayo Clinic (of note, the reporting strategy differed between the two studies), ${ }^{1,14} 0.034 \%$ between 1988 and 1992 at the University of Chicago, ${ }^{15}$ $0.12 \%$ in 2012 at the University of Alabama (UAB), ${ }^{16}$ and $0.07 \%$ between 2011 and 2013 at New York University. ${ }^{13}$ The Mayo Clinic and UAB anesthesia departments each performed a quality improvement (QI) corneal abrasion prevention program; incidence of perioperative corneal injury decreased from 0.15 to $0.047 \%$ at Mayo and from 0.12 to $0.009 \%$ at $\mathrm{UAB} .{ }^{16}$ No such reports exist in the ophthalmology literature, with primarily anesthesiologists taking the lead to develop their own corneal injury prevention and management plans.
The purpose of the current study was to investigate the impact of an ophthalmology resident-led QI initiative aimed to decrease the incidence of perioperative corneal injury at an academic medical center.

\section{Methods}

The study was granted an exemption from the Institutional Review Board of the Penn State Health Milton S. Hershey Medical Center. A retrospective chart review was conducted of all surgical cases performed during 6 months prior to, and 6 months after, implementation of an ophthalmology resident-led QI initiative at Penn State Milton S. Hershey Medical Center. The QI initiative consisted of a lecture and distribution of educational materials to anesthesia providers based on a Mayo Clinic Model. ${ }^{1}$ The lecture focused on perioperative corneal injury awareness, understanding of risk factors, and presentation of an algorithm designed to prevent perioperative corneal injury. Anesthesia providers were instructed by the ophthalmology chief resident how and when to tape the eyes shut during anesthesia induction, with emphasis on ensuring full eyelid closure over the globe to avoid corneal drying. Use of eye ointment was recommended for those cases involving previously reported risk factors for corneal injury, including operative times $>90$ minutes, head and neck cases, prone or lateral patient positioning, or the presence of proptosis or lagophthalmos. Data collected through the chart review included type of surgical case, presence of diabetes mellitus or thyroid disease (which are risk factors for preexisting corneal pathology), patient age, gender, patient positioning (supine, prone, or lateral), level of primary anesthesia provider training (all cases were supervised by an attending anesthesiologist) (resident in postgraduate year [PGY] 1, 2, 3, or 4, certified registered nurse anesthetist [CRNA]), or fellow), length of surgical case in minutes, surgical service, type of anesthesia, and type (if any) of perioperative eye injury.

\section{Statistical Methods}

All variables were summarized prior to analysis to check for errors and assess their distributions. Duration of surgery was grouped into durations of $<90,90$ to 180 , or $>180$ minutes based on the model by Batra and Bali. ${ }^{11}$ Logistic regression was performed to evaluate potential risk factors for perioperative corneal injury including several patient and surgical factors: age ( $<25,25-<50,50-<65$, and $\geq 65$ years), gender, type of anesthesia (general anesthesia vs. other), type of surgery (head and neck surgery vs. other), surgical service (general surgery vs. other subspecialty services), diabetes mellitus, thyroid disease, patient positioning (supine, prone, 
or lateral), level of anesthesia provider training (PGY 1, 2, 3, or 4, CRNA, fellow), length of case in minutes, and type (if any) of perioperative corneal injury (abrasion, exposure, vs. toxic). This analysis of potential predictors of perioperative corneal injury was adjusted for the phase of initiative by including it as a covariate in the logistic regression model. The incidence of perioperative corneal injury was compared pre- versus post-QI initiative using logistic regression and was adjusted for age and gender in addition to the significant variables from the analysis of potential risk factors: general surgery, patient position, and duration of surgery. Odds ratios (ORs) and 95\% confidence intervals (CIs) are used to quantify the magnitude and direction of significant associations for the logistic regression analyses. Statistical significance was set at 0.05. All analyses were performed using SAS software version 9.4 (SAS Institute, Cary, NC).

\section{Results}

\section{Effectiveness of QI Initiative}

The incidence of perioperative corneal injury decreased from $0.37 \%$ ( 36 of 9,745 cases) pre-initiative to $0.19 \%$ (19 of 9,991 cases) post-initiative $(p=0.012)$. There was no significant difference pre- vs. post-initiative in the proportion of corneal injury cases resulting from abrasion (vs. exposure) $(p=0.909$; - Table 1). Of note, there were no reported cases of toxic keratopathy in either the pre- or post-initiative phases.

\section{Assessment of Risk Factors for Perioperative Corneal Injury}

Analysis of risk factors, with consideration of initiative phase as a covariate, demonstrated an increased risk of perioperative corneal injury associated with non-head and neck cases (OR $=0.32$, 95\% CI 0.11-0.87; $p=0.026$ ), supine compared with lateral patient positioning $(\mathrm{OR}=0.13,95 \%$ CI $0.07-0.23$; $p=0.006)$, prone compared with lateral positioning $(\mathrm{OR}=0.25$, $95 \%$ CI $0.09-0.67 ; p<0.001$ ), surgery performed by the general surgery service (OR 6.50, 95\% CI 2.39-24.76, $p<0.001$ ), and longer surgical time. A significantly higher incidence of perioperative corneal injury was observed when comparing cases of 90 to 180 minutes' duration to cases of $<90$ minutes' duration $(\mathrm{OR}=4.18,95 \%$ CI 1.43-12.18; $p=0.009),>180$ minutes to $<90$ minutes $(\mathrm{OR}=8.56,95 \% \mathrm{CI} 3.01-24.32 ; p<0.001)$, and $>180$ minutes to 90 to 180 minutes $(\mathrm{OR}=2.05,95 \% \mathrm{CI}$ 1.17-3.58; $p<0.001$; - Table 2). There was no increased risk of corneal injury with regard to the following factors: age, female versus male gender $(\mathrm{OR}=0.94,95 \% \mathrm{CI} 0.55-1.59$, $p=0.809$ ), general anesthesia versus regional/conscious sedation/other $(\mathrm{OR}=0.77,95 \% \mathrm{CI} 0.35-2.02, p=0.639)$, diagnosis of diabetes mellitus ( $\mathrm{OR}=0.98,95 \% \mathrm{CI} 0.42-2.30$; $p=0.967)$ or thyroid disease $(\mathrm{OR}=1.72,95 \% \mathrm{CI} 0.78-3.81$; $p=0.182$ ), and level of the anesthesia provider (-Table 2 ).

The general surgery service was then reviewed for the incidence of corneal injury in each separate general surgery category (colorectal surgery, cardiac surgery, emergency general surgery, general surgery, neurosurgery, orthopedics, plastic surgery, minimally invasive surgery, surgical oncology, transplant surgery, trauma surgery, urology, vascular surgery, and pediatric general surgery); there was a significantly higher incidence and odds of corneal injury in the vascular surgery group versus the other groups even after adjustment for other significant risk factors for perioperative corneal eye injury such as surgical time and positioning (1.10\% vs. $0.31 \%, \mathrm{OR}=3.70, \mathrm{CI} 1.57-8.73, p=0.003)$.

\section{Discussion}

In the current study, an ophthalmology resident-led educational initiative for anesthesia providers on perioperative corneal injury awareness, risk factors of perioperative corneal injury, and strategies to prevent perioperative corneal injury was associated with a significant decrease in the incidence of perioperative corneal injury (-Fig. 1, - Table 1). Similar to previous studies that evaluated risk factors for perioperative corneal injury, we found that longer duration of surgery poses an increased risk for corneal injury. ${ }^{4,17}$ This is likely due to the additional time during which the patient is exposed to such effects of anesthesia as decreased tear film production and absence of the Bell's phenomenon. ${ }^{4,5}$ We did not find a time point at which that risk plateaued; rather, with each 90minute unit increase in length of surgery, the risk of injury also increased. In our study, there was no significant difference in injury rates among the various levels of anesthesia providers. A previous study showed an increased incidence of perioperative corneal injury with student nurse anesthetists compared with residents or CRNAs ${ }^{1}$; however, our institution does not have such trainees and we found no increased risk associated with earlier trainee level of resident physician.

As in previous studies, we found an increased risk of perioperative corneal injury in patients who were placed in the lateral or prone positions compared with the supine position..$^{4,12,14}$ To place a patient in a lateral or prone position often requires rolling the patient after anesthetics have been administered. The process of moving the patient and location of the face in a compromising position may be why lateral and prone positioning demonstrate an increased risk. In

Table 1 Pre- and post-initiative corneal injury data comparison

\begin{tabular}{|l|l|l|l|l|}
\hline & Pre-initiative & Post-initiative & Odds ratio (95\% CI) & $p$-Value \\
\hline Rate of perioperative corneal injury after nonocular surgery & $0.37 \%$ & $0.19 \%$ & $0.49(0.28-0.85)$ & 0.012 \\
\hline $\begin{array}{l}\text { Proportion of perioperative corneal injury } \\
\text { cases resulting from abrasion (vs. exposure) }\end{array}$ & $53.66 \%$ & $52.17 \%$ & $0.94(0.34-2.62)$ & 0.909 \\
\hline
\end{tabular}

Abbreviation: $\mathrm{Cl}$, confidence interval.

Note: Odds ratios and $p$-values from logistic regression.

${ }^{a}$ Adjusted for age, gender, general surgery, patient position, and duration of surgery. 
Table 2 Risk factor analysis for pre- and post-initiative cases of perioperative corneal injury after nonocular surgery

\begin{tabular}{|c|c|c|c|c|}
\hline & Control cases $(n=20,187)$ & Injury cases $(n=55)$ & Odds ratio $(95 \% \mathrm{Cl})$ & $p$-Value \\
\hline \multicolumn{5}{|l|}{ Age $(y)$} \\
\hline$<25$ & $4,921(99.9 \%)$ & $7(0.1 \%)$ & $0.55(0.18-1.50)$ & 0.286 \\
\hline $25-<50$ & $5,334(99.7 \%)$ & $18(0.3 \%)$ & $1.33(0.61-3.03)$ & 0.561 \\
\hline $50-<65$ & 4,991 (99.6\%) & $18(0.4 \%)$ & $1.41(0.64-3.21)$ & 0.460 \\
\hline$\geq 65$ & $4,691(99.7 \%)$ & $12(0.3 \%)$ & 1.0 & \\
\hline \multicolumn{5}{|l|}{ Gender } \\
\hline Female & 10,289 (99.7\%) & $27(0.3 \%)$ & $0.94(0.55-1.59)$ & 0.809 \\
\hline Male & $9,898(99.7 \%)$ & $28(0.3 \%)$ & 1.0 & \\
\hline \multicolumn{5}{|c|}{ Diabetes mellitus } \\
\hline Yes & $2,255(99.7 \%)$ & $6(0.3 \%)$ & $0.98(0.42-2.30)$ & 0.967 \\
\hline No & 17,932 (99.7\%) & $49(0.3 \%)$ & 1.0 & \\
\hline \multicolumn{5}{|l|}{ Thyroid disease } \\
\hline Yes & $1,579(99.6 \%)$ & $7(0.4 \%)$ & $1.72(0.78-3.81)$ & 0.182 \\
\hline No & $18,608(99.7 \%)$ & $48(0.3 \%)$ & 1.0 & \\
\hline \multicolumn{5}{|l|}{ General surgery } \\
\hline Yes & $13,403(99.6 \%)$ & $51(0.4 \%)$ & $6.50(2.39-24.76)$ & $<0.001$ \\
\hline No & $6,784(99.9 \%)$ & $4(0.1 \%)$ & 1.0 & \\
\hline \multicolumn{5}{|c|}{ General anesthesia } \\
\hline Yes & 17,905 (99.8\%) & $48(0.3 \%)$ & $0.77(0.35-2.02)$ & 0.639 \\
\hline No & $2,023(99.7 \%)$ & $7(0.3 \%)$ & 1.0 & \\
\hline \multicolumn{5}{|c|}{ Head and neck case } \\
\hline Yes & 4,008 (99.9\%) & $4(0.1 \%)$ & $0.32(0.11-0.87)$ & 0.026 \\
\hline No & $16,179(99.7 \%)$ & $51(0.3 \%)$ & 1.0 & \\
\hline \multicolumn{5}{|l|}{ Positioning $^{a}$} \\
\hline Lateral & $1,331(98.6 \%)$ & $19(1.4 \%)$ & 1.0 & \\
\hline Prone & 1,393 (99.6\%) & $5(0.4 \%)$ & $0.25(0.09-0.67)$ & 0.006 \\
\hline Supine & 16,998 (99.8\%) & $31(0.2 \%)$ & $0.13(0.07-0.23)$ & $<0.001$ \\
\hline \multicolumn{5}{|c|}{ Anesthesia provider $^{b}$} \\
\hline PGY1 & 2,606 (99.6\%) & $11(0.4 \%)$ & $1.67(0.86-3.23)$ & 0.131 \\
\hline PGY2 & $5,423(99.8 \%)$ & $9(0.2 \%)$ & $0.54(0.26-1.10)$ & 0.090 \\
\hline PGY3 & $4,392(99.7 \%)$ & $15(0.3 \%)$ & $1.32(0.73-2.40)$ & 0.357 \\
\hline PGY4 & 2,185 (99.8\%) & $5(0.2 \%)$ & $0.81(0.32-2.02)$ & 0.644 \\
\hline CRNA & $5,213(99.7 \%)$ & $15(0.3 \%)$ & $1.13(0.63-2.05)$ & 0.681 \\
\hline Fellow & $458(100.0 \%)$ & $0(0.0 \%)$ & $\mathrm{N} / \mathrm{A}$ & 0.283 \\
\hline \multicolumn{5}{|l|}{ Duration $^{c}$} \\
\hline$<90 \min$ & $6,579(99.9 \%)$ & $4(0.1 \%)$ & 1.0 & \\
\hline $90-180 \mathrm{~min}$ & $8,043(99.7 \%)$ & $21(0.3 \%)$ & $4.18(1.43-12.18)$ & 0.009 \\
\hline$>180 \mathrm{~min}$ & $5,523(99.5 \%)$ & $30(0.5 \%)$ & $8.56(3.01-24.32)$ & $<0.001$ \\
\hline
\end{tabular}

Abbreviations: $\mathrm{Cl}$, confidence interval; CRNA, certified registered nurse anesthetist; OR, odds ratio; PGY, postgraduate year.

Note: Odds ratios and $p$-values from a logistic regression adjusted for the phase of the initiative. Exact logistic regression used as needed.

${ }^{\mathrm{a}} \mathrm{OR}$ for prone versus supine is 1.95 (0.77, 5.02), $p=0.167$.

${ }^{\mathrm{b} C o m p a r i s o n}$ of each provider level individually to all other levels combined to determine if there was a significant difference between groups. ${ }^{c} \mathrm{OR}$ for $>180$ minutes versus $90-180$ minutes is 2.05 (1.17-3.58), $p<0.001$.

contrast to other published reports, ${ }^{1,15}$ our study identified an increased risk of perioperative corneal injury in nonhead and neck cases. A potential explanation for this increased risk may be a decreased awareness of contact with the patient's face, including the eye, if the eye is not in direct view during the procedure. Also, in contrast to a previous report, ${ }^{1}$ our study did not identify thyroid disease as a significant predictor of perioperative corneal injury. This may be due, at least 


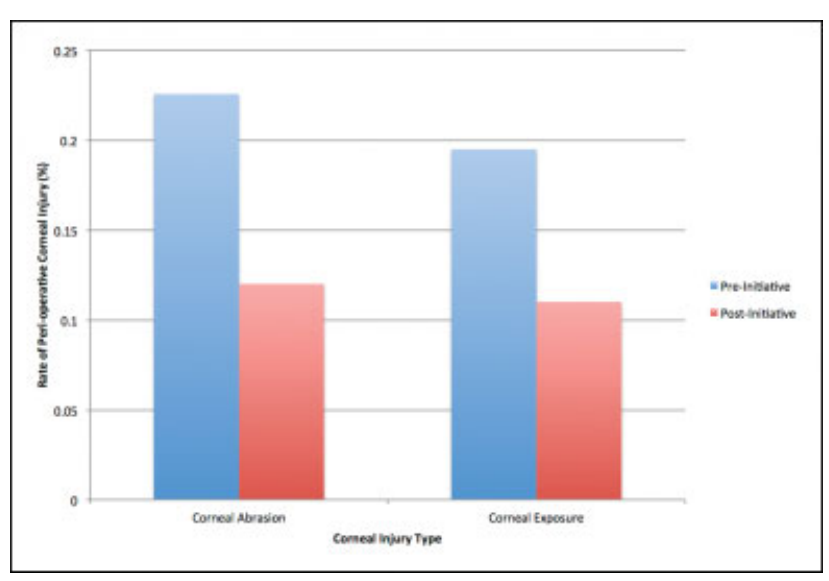

Fig. 1 Decreased incidence of perioperative corneal injury after ophthalmology resident-led quality improvement initiative.

in part, to the fact that the previously published study ${ }^{1}$ isolated Graves' disease as a specific diagnosis. Due to the nature of our electronic medical records database, we were unable to distinguish Graves' disease from other thyroid diagnoses.

To our knowledge, and based on a computerized search of the PubMed database, one other study looked for a difference in risk of corneal injury among surgical services. The latter study, conducted at New York University, did not find a significant difference. ${ }^{13}$ In contrast, our study identified a significantly higher rate of perioperative corneal injury associated with vascular surgery compared with other surgical services. Although the vascular surgery group had a significantly higher proportion of patients with such previously identified risk factors for corneal injury such as supine position and longer surgical time, vascular surgery had an independently significant effect on corneal injury risk even after adjustment for the previously identified risk factors. Further study of this finding is warranted.

One limitation of the current study is the retrospective nature of the chart review. However, this applied equally to the pre- and post-initiative phases. Another limitation of our study is the relatively short (6 months) follow-up period of post-initiative data collection. The study from Mayo Clinic initially included 16 months of follow-up post-initiative and then increased follow-up to 31 months, and UAB included a follow-up of 45 months post-initiative. ${ }^{1,16}$ Our study was designed to fit within one training year to allow for a resident-run initiative. Further research is warranted to investigate longer-term outcomes of an ophthalmology resident-led program designed to decrease the incidence of perioperative corneal injury.

In sum, our findings show that an ophthalmology resident-led educational initiative to anesthesiology providers was associated with a significant decrease in the incidence of perioperative corneal injury. We hope that this QI initiative may inspire other ophthalmology practices to take the lead in perioperative corneal injury awareness and prevention.

\section{Conclusion}

An ophthalmology resident-led educational initiative to anesthesiology providers was associated with a significant decrease in the incidence of perioperative corneal injury.

\section{Funding}

E.L. received support from the National Institutes of Health, http://dx.doi.org/10.13039/100000002, UL1 TR00 2014. Funding for statistical analysis was provided by the CTSA grant and the National Institutes of Health (NIH).

\section{Conflict of Interest}

E.L. reports grants from National Institutes of Health (NIH), during the conduct of the study.

\section{References}

1 Martin DP, Weingarten TN, Gunn PW, et al. Performance improvement system and postoperative corneal injuries: incidence and risk factors. Anesthesiology 2009;111(02):320-326

2 Gild WM, Posner KL, Caplan RA, Cheney FW. Eye injuries associated with anesthesia. A closed claims analysis. Anesthesiology 1992;76(02):204-208

3 Posner KL, Lee L. Anesthesia malpractice claims associated with eye surgery and eye injury: highlights from the anesthesia closed claims project data request service. ASA Newsl 2014;78(11):28-30

4 White E, Crosse MM. The aetiology and prevention of perioperative corneal abrasions. Anaesthesia 1998;53(02):157-161

5 Brock-Utne JG, Botz G, Jaffe RA. Perioperative corneal abrasions. Anesthesiology 1992;77(01):221

6 Liu H-Y, Yeh PT, Kuo KT, et al. Toxic keratopathy following the use of alcohol-containing antiseptics in nonocular surgery. JAMA Ophthalmol 2016;134(04):449-452

7 Fraser S. Corneal abrasion. Clin Ophthalmol 2010;4:387-390

8 Wilson SA, Last A. Management of corneal abrasions. Am Fam Physician 2004;70(01):123-128

9 Carter JA, Utiger RD. The ophthalmopathy of Graves' disease. Annu Rev Med 1992;43:487-495

10 Cousen P, Cackett P, Bennett H, Swa K, Dhillon B. Tear production and corneal sensitivity in diabetes. J Diabetes Complications 2007;21(06):371-373

11 Batra YK, Bali IM. Corneal abrasions during general anesthesia. Anesthesia and Analgesia 1977;56(03):363-365

12 Yu HD, Chou AH, Yang MW, Chang CJ. An analysis of perioperative eye injuries after nonocular surgery. Acta Anaesthesiol Taiwan 2010;48(03):122-129

13 Carniciu AL, Fazzari MJ, Tabibian P, et al. Cornea abrasion following anaesthesia for non-ocular surgical procedures: a case-controlled study. J Perioper Pract 2017;27(11):247-253

14 Deljou A, Weingarten TN, Mahr MA, Sprung J, Martin DP. Postoperative corneal injuries: Incidence and risk factors. Anesth Analg 2019;129(03):737-742

15 Roth S, Thisted RA, Erickson JP, Black S, Schreider BD. Eye injuries after nonocular surgery. A study of 60,965 anesthetics from 1988 to 1992. Anesthesiology 1996;85(05):1020-1027

16 Vetter TR, Ali NM, Boudreaux AM. A case-control study of an intraoperative corneal abrasion prevention program: holding the gains made with a continuous quality improvement effort. Jt Comm J Qual Patient Saf 2012;38(11):490-496

17 Batra YK, Bali IM. Corneal abrasions during general anesthesia. Anesth Analg 1977;56(03):363-365 